\title{
ESTILOS DE APRENDIZAGEM E HIERARQUIA DE NECESSIDADES NO PLANEJAMENTO EDUCACIONAL REMOTO EM TEMPOS DE PANDEMIA
}

\author{
Igor luco Castro-Silva ${ }^{1}$, Jacques Antonio Cavalcante Maciel ${ }^{2}$, Lana Karine Araújo ${ }^{3}$
}

\section{RESUMO}

A pandemia por COVID-19 desafia a educação superior para a ensinagem remota. O objetivo do estudo foi relatar o diagnóstico situacional deste contexto educacional. Estudantes de Odontologia e Psicologia $(n=96)$ preencheram dois questionários de estilos de aprendizagem VARK e hierarquia de necessidades de Maslow. Houve, no curso de Odontologia, um perfil cinestésico e maiores pontuações na dimensão fisiológica, segurança e estima, em comparação ao estilo auditivo e menor acesso à internet do curso de Psicologia. Recursos multimídia tiveram boa aceitabilidade, e tecnologias digitais, incluindo vídeos e podcasts, sugeriram maior envolvimento dos discentes. A oferta assíncrona de conteúdos poderia minimizar limitações de acessibilidade digital. Os estilos de aprendizagem VARK e a hierarquia de necessidades humanas de Maslow podem contribuir positivamente para o planejamento pedagógico de atividades remotas em estudantes da Saúde.

Palavras-chave: Aprendizagem. Educação a distância. Educação superior.

\section{Como citar este documento - ABNT}

CASTRO-SILVA, Igor luco; MACIEL, Jacques Antonio Cavalcante; ARAÚJO, Lana Karine. Estilos de aprendizagem e hierarquia de necessidades no planejamento educacional remoto em tempos de pandemia. Revista Docência do Ensino Superior, Belo Horizonte, v. 10, e024677, p. 1-16, 2020. DOI: https://doi.org/10.35699/2237-5864.2020.24677.

Recebido em: $14 / 08 / 2020$ Aprovado em: 05/11/2020 Publicado em: $14 / 12 / 2020$

\footnotetext{
${ }^{1}$ Universidade Federal do Ceará (UFC), Sobral, CE, Brasil. ORCID ID: https://orcid.org/0000-0003-4815-6357. E-mail: igor.iuco@sobral.ufc.br.

2 Universidade Estadual Vale do Acaraú (UVA) e Universidade Federal do Ceará (UFC), Sobral, CE, Brasil. ORCID ID: https://orcid.org/0000-0002-2293-8433. E-mail: jacques.maciel@sobral.ufc.br.

${ }^{3}$ Universidade Federal do Ceará (UFC), Sobral, CE, Brasil.

ORCID ID: https://orcid.org/0000-0003-2200-8313. E-mail: lanakarine1380@gmail.com.
} 


\section{ESTILOS DE APRENDIZAJE Y JERARQUÍA DE NECESIDADES EN LA PLANIFICACIÓN EDUCATIVA REMOTA EN TIEMPOS DE PANDEMIA}

\section{RESUMEN}

La pandemia COVID-19 desafía a la educación superior para enseñanza-aprendizaje remoto. El objetivo del estudio fue reportar el diagnóstico situacional de este contexto educativo. Estudiantes de Odontología y Psicología(n=96) completaron dos cuestionarios de estilos de aprendizaje de VARK y la jerarquía de necesidades de Maslow. Hubo un perfil cinestésico en la carrera de Odontología y puntuaciones más altas en la dimensión fisiológica, seguridad y estima, en comparación con estilo auditivo y menor acceso a internet en la carrera de Psicología. Recursos multimedia tuvieron buena aceptación y tecnologías digitales, incluidos videos y podcasts, sugirieron mayor participación de los estudiantes. Oferta de contenido asincrónico podría minimizar limitaciones de accesibilidad digital. Estilos de aprendizaje de VARK y la jerarquía de necesidades humanas de Maslow pueden contribuir positivamente a la planificación pedagógica de actividades remotas en los estudiantes del área de la Salud.

Palabras clave: Aprendizaje. Educación a distancia. Educación superior.

\section{LEARNING STYLES AND HIERARCHY OF NEEDS IN THE REMOTE EDUCATIONAL PLANNING IN PANDEMIC TIMES}

\section{ABSTRACT}

The COVID-19 pandemic challenges higher education for remote teaching. The aim of this study was to report the situational diagnosis in this educational context. Dentistry and Psychology students $(n=96)$ completed two questionnaires on VARK learning styles and Maslow's hierarchy of human needs. In the Dentistry course there was a kinesthetic profile and higher scores in the physiological dimension, security and esteem, in comparison to the auditory style and less internet access of the Psychology course. Multimedia resources had good acceptability, and digital technologies, including videos and podcasts, suggested greater involvement of students. The asynchronous content offering could minimize limitations on digital accessibility. VARK learning styles and Maslow's hierarchy of human needs can positively contribute to the pedagogical planning of remote activities in Health students.

Keywords: Learning. Distance learning. Higher education. 
Igor luco Castro-Silva, Jacques Antonio Cavalcante Maciel, Lana Karine Araújo

\section{INTRODUÇÃO}

A pandemia por COVID-19 alterou drasticamente as relações humanas, impondo uma rotina de controle sanitário rígido e o distanciamento social horizontal na maioria dos países do mundo, visto que a prevenção física à contaminação constitui a melhor forma de combate até o momento (MACIEL; CASTRO-SILVA; FARIAS, 2020). Na educação superior, a modalidade presencial é base para o desenvolvimento de competências cognitivas, psicomotoras e atitudinais de estudantes de cursos de graduação da área de Saúde (CASTRO-SILVA; GUERRERO; MACIEL, 2018; DIAS et al., 2020; MACIEL et al., 2016). Neste cenário adverso, o processo de ensino-aprendizagem, ou ensinagem, só é possível na forma remota (ZAYAPRAGASSARAZAN, 2020). Formatos substitutivos têm sido dialogados, mas o curto tempo para enfrentamento ainda não provê todas as respostas necessárias (BRASIL, 2020). Teleaulas, textos digitais, e-books, vídeos complementares, áudios, simulações online, jogos eletrônicos e dispositivos adaptativos elencam os recursos educacionais úteis em ambientes virtuais (ABED, 2019; SILVA; AMARO; MATTAR, 2019).

O Brasil é heterogêneo no uso da Educação a Distância (EaD), seja no formato integral ou parcial, cujas instituições estão concentradas na região Sudeste, em equivalência ao dobro da Nordeste e ao sêxtuplo da Norte, e nas capitais, que chegam ao dobro do quantitativo presente no interior do estado (ABED, 2019). Apesar de o público-alvo ser adulto jovem, na faixa de 21 a 40 anos, a EaD na educação superior é maior em cursos de lato sensu, não sendo um perfil comum da graduação, seja na modalidade bacharelado, licenciatura ou tecnológica (ABED, 2019; MACIEL; JIMENEZ; CASTRO-SILVA, 2020). A oferta de graduações com EaD parcial na área de Ciências da Saúde é baixa, chegando a um terço das Ciências Sociais ou Humanas (ABED, 2019). A evasão geral dos cursos com EaD é alta, entre $26 \%$ e $50 \%$, frente aos cursos presenciais, entre $0 \%$ e $5 \%$, o que torna importante buscar estratégias para fidelização do estudante (ABED, 2019).

O preparo de docentes e instituições para promoverem condições de ensino remoto, bem como a aderência de estudantes da modalidade presencial às atividades on-line, constitui ainda questões obscuras, sendo preocupação legítima para atingir uma aprendizagem satisfatória (PANÚNCIO-PINTO; TRONCON, 2014; ZAYAPRAGASSARAZAN, 2020). Fatores intrínsecos e extrínsecos do público estudantil poderiam refletir sobre o ambiente educacional e, neste momento de pandemia, é estratégico o seu mapeamento para buscar metodologias e recursos mais significativos para a ensinagem (CASTRO-SILVA; GUERRERO; MACIEL, 2018; TRONCON, 2014).

Segundo Schmitt e Domingues (2016), não existe uma abordagem universal para definir tipos de aprendizagem, sendo publicados diferentes sistemas classificatórios na literatura, incluindo: modelo de Dunn-Dunn, em 1978, Gregorc, em 1979, Kolb, em 1984, Felder- 
Silverman, em 1988, e VARK, criado por Flemming, em 1992. Este modelo mapeia as preferências de comportamento de estudantes, em quatro canais de aprendizado, pelo acrônimo em inglês VARK: visual (visual), aural (auditivo), reading/writing (leitura/escrita) e kinesthetic (cinestésico). A simplicidade de aplicação e a rapidez para obtenção e interpretação de resultados frente aos demais modelos são vantagens do VARK para o planejamento educacional de urgência ou de grandes grupos de estudantes (SCHMITT; DOMINGUES, 2016).

Panúncio-Pinto e Troncon (2014) reforçam que o cenário é tão importante quanto os atores no diagnóstico situacional do ambiente educativo. Troncon (2014) revisou a teoria de motivação humana de Maslow, publicada em 1954, publicou uma teoria de motivação humana que inspirou a construção da pirâmide de necessidades, de primárias a secundárias (TRONCON, 2014). Dentro dos conceitos de psicologia humanista, a pirâmide de Maslow teve reforço da teoria freudiana das pulsões e críticas diante de reações heterogêneas dos indivíduos ao mesmo estímulo, do prazer à insatisfação, embora preserve sua importância organizacional de recursos humanos até o momento (PANÚNCIO-PINTO; TRONCON, 2014; TRONCON, 2014).

Dessa forma, o objetivo deste relato foi compartilhar a experiência com aplicação de questionários de estilos de aprendizagem e de hierarquia de necessidades para o planejamento de ensinagem remota em diferentes cursos da Saúde na pandemia.

\section{METODOLOGIA}

Este estudo foi aprovado pelo Comitê de Ética em Pesquisa da Universidade Estadual Vale do Acaraú sob o parecer n. 4.009.393/2020 e esteve em conformidade com as regulamentações ético-legais vigentes em pesquisa com seres humanos no Brasil.

O cenário deste relato de experiência foi o campus da Universidade Federal do Ceará sediado em Sobral, cidade do interior da região norte do estado, durante o semestre 2020/1. O público-alvo foi composto por 96 estudantes de graduação, sendo 49 do primeiro período em Odontologia na disciplina teórico-prática Concepção e Formação do Corpo Humano I e 47 do terceiro período em Psicologia na disciplina teórica Fisiologia Geral e Psiconeurofisiologia.

O primeiro semestre letivo de 2020 teve o seu calendário adaptado. Atividades presenciais foram conduzidas de 17 de fevereiro a 16 de março, até o momento de declaração institucional do estado de pandemia pela COVID-19. Atividades remotas voluntárias, em caráter de urgência e sem caráter avaliativo, prosseguiram entre 17 de março e 19 de julho. O retorno das aulas oficiais previu atividades remotas obrigatórias para componentes teóricos, de 20 de julho a 22 de outubro de 2020, cumprindo, assim, 100 dias letivos, 
conforme preconizado pela Lei n. 9.394/1996 (BRASIL, 1996) e dentro da flexibilização por calamidade pública publicada pela Portaria n. 544/2020 (BRASIL, 2020). Durante a fase de distanciamento social e antes do retorno oficial, entre 1 e 8 de julho de 2020, todos os estudantes matriculados nas duas disciplinas foram convidados a participar voluntariamente de duas atividades diagnósticas, envolvendo o preenchimento de um questionário de estilos de aprendizagem e outro de hierarquia de necessidades humanas.

Para investigar os estilos de aprendizagem dos estudantes, foi aplicado o questionário psicométrico padronizado VARK na versão traduzida para o português, contendo 16 perguntas fechadas, com possibilidade de uma ou mais respostas objetivas. Tais respostas especificaram o padrão visual $(V)$, auditivo $(A)$, de leitura ou escrita $(R)$ ou cinestésico $(K)$ (PROBST, 2020). Este instrumento diagnostica um ou mais estilos preferenciais de aprendizagem por estudante. As maiores frequências unimodais independentes de cada categoria foram expressas em dados relativos, para cada curso em análise.

Um segundo questionário foi desenvolvido pelos próprios autores, dialogado junto à Comissão de Acompanhamento Pedagógico e Discente do curso de Odontologia da própria instituição de ensino. O propósito do instrumento simplificado foi o levantamento rápido de condições influenciadoras na participação das aulas remotas, baseado na pirâmide de hierarquia das necessidades humanas de Maslow, que engloba cinco dimensões (TRONCON, 2014). Dentro das dimensões propostas, existem as categorias de dimensões primárias e secundárias. Constituem dimensões primárias: fisiológica (condições de sobrevivência ou homeostasia) e de segurança (condições de segurança do corpo, de recursos e da propriedade). Já as dimensões secundárias são compostas por: social (vínculos familiares, de amizade e acadêmicos), de estima (relações de autoestima, confiança e respeitabilidade) e de realização pessoal (capacidade de solução de problemas, criatividade e aceitação dos fatos). Apesar de o acesso à internet ser uma forma de comunicação, de início configurável dentro da dimensão social, os autores optaram pela classificação na dimensão de segurança, pelo entendimento da importância primária na viabilidade físico-tecnológica da educação remota. $O$ instrumento de rastreio elaborado, contendo todas as perguntas propostas, bem como suas respostas aos itens seguindo uma escala Likert de 0 a 4 , está disponível no Quadro 1. 


\begin{tabular}{|c|}
\hline Dimensão 1: Fisiológica \\
\hline $\begin{array}{l}\text { 1.1 Tem dormido bem? } \square 0 \square 1 \square 2 \square 3 \square 4 \\
\text { 1.2 Tem se alimentado bem? } \square 0 \square 1 \square 2 \square 3 \square 4\end{array}$ \\
\hline Dimensão2: Segurança \\
\hline $\begin{array}{l}\text { 2.1 Tem o mesmo poder aquisitivo de antes da pandemia? } \square 0 \square 1 \square 2 \square 3 \square 4 \\
\text { 2.2 Tem condições satisfatórias de moradia? } \square 0 \square 1 \square 2 \square 3 \square 4 \\
\text { 2.3 Tem condições satisfatórias de acesso à internet? } \square 0 \square 1 \square 2 \square 3 \square 4\end{array}$ \\
\hline Dimensão 3: Social \\
\hline $\begin{array}{l}\text { 3.1 Mantém contato com círculo familiar e de amigos? } \square 0 \square 1 \square 2 \square 3 \square 4 \\
\text { 3.2 Mantém contato com professores e colegas de faculdade? } \square 0 \square 1 \square 2 \square 3 \square 4\end{array}$ \\
\hline Dimensão 4: Estima \\
\hline $\begin{array}{l}\text { 4.1 Se sente satisfeito com sua aparência? } \square 0 \square 1 \square 2 \square 3 \square 4 \\
\text { 4.2 Realiza atividades de vida diária (escova dentes, banho, troca roupa)? } \square 0 \square 1 \square 2 \square 3 \square 4\end{array}$ \\
\hline Dimensão 5: Realização pessoal \\
\hline $\begin{array}{l}\text { 5.1 Se sente capaz de realizar as atividades remotas propostas na faculdade? } \square 0 \square 1 \square 2 \square 3 \square 4 \\
\text { 5.2 Se sente feliz no curso de graduação escolhido? } \square 0 \square 1 \square 2 \square 3 \square 4\end{array}$ \\
\hline $\begin{array}{l}\text { Legenda: 0-Nenhum dia do distanciamento, 1-Algumas vezes no distanciamento, 2-Algumas vezes } \\
\text { no mês, 3-Algumas vezes na semana e } 4 \text {-Todos os dias. }\end{array}$ \\
\hline
\end{tabular}

Quadro 1 - Questionário para levantamento de necessidades de estudantes na pandemia Fonte: elaborado pelos autores (2020).

Foi delineado um plano pedagógico de emergência, pautando, na metodologia de EaD, a divisão de unidades bimestrais da ementa em três blocos (conteúdo, interação e avaliação), através de recursos síncronos e assíncronos. Os recursos selecionados para a ensinagem remota consideraram o diagnóstico dos múltiplos estilos de aprendizagem presentes, com ênfase nos modelos mais prevalentes nos cursos, e da hierarquia das necessidades, permitindo flexibilização temporal de atividades e inclusão digital de todos os estudantes na pandemia.

Para o conteúdo, foram disponibilizados como recursos assíncronos no sistema integrado de gestão acadêmica, ao qual todos os alunos matriculados têm acesso: 1) slides com conteúdo das disciplinas; 2) videoaulas gravadas pelo professor, com duração de 30 a 60 minutos; 3) vídeos temáticos complementares; 4) podcasts, com duração de 10 minutos cada, com perguntas norteadoras e problematizações dos conteúdos apresentados; 5) bibliografia complementar para leitura, na forma de artigos atuais e contextualizados. Para a interação e avaliação formativa, foram disponibilizados recursos síncronos, através de teleconferências por Google Meet, direcionadas como plantão de dúvidas e instruções gerais das disciplinas, que também foram gravadas e disponibilizadas no sistema acadêmico para posterior visualização assíncrona. Avaliações somativas contaram com questões de múltipla escolha e discursivas, com prazo de entrega via sistema acadêmico. 
A aderência dos estudantes dos cursos, durante os meses de abril a julho, aos recursos digitais foi contabilizada pelos registros eletrônicos de visualização de conteúdo assíncrono no sistema acadêmico ou durante a chamada virtual, no caso da teleconferência síncrona (FIGURA 1). Os dados foram expressos em percentagem, expressando a média do número de estudantes por turma ao longo do período que fizeram uso de algum dos recursos propostos.

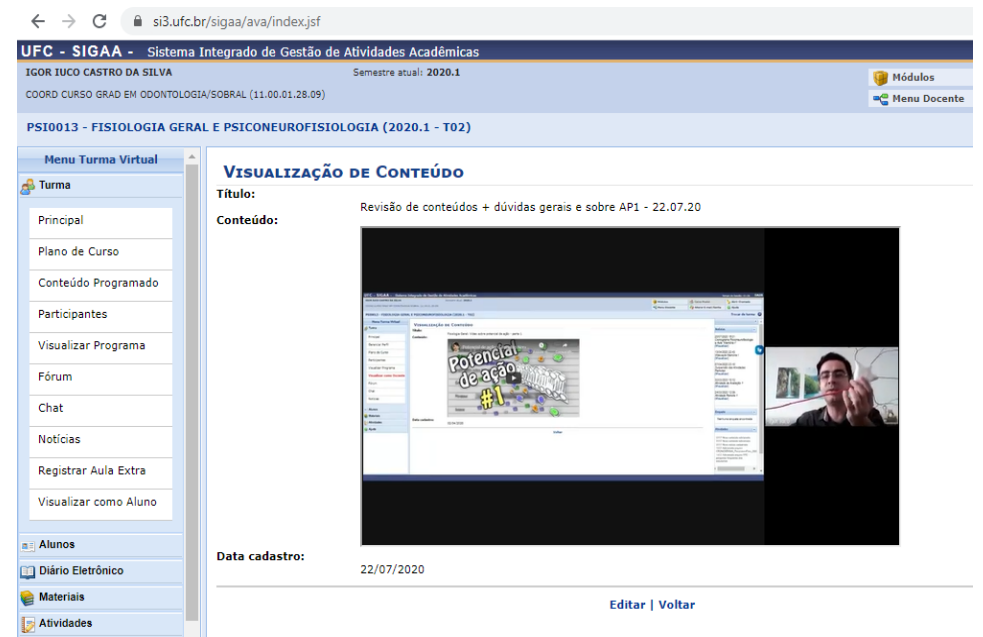

Figura 1 - Recursos virtuais no sistema acadêmico Fonte: elaborada pelos autores (2020).

Para atingir a percepção dos estudantes, foi lançada uma enquete aplicada no sistema acadêmico em julho, com a pergunta norteadora: "Descreva em uma palavra, à sua escolha, o que tem representado para você a experiência nessa disciplina on-line". Os resultados foram agrupados e expressos na forma de nuvem de palavras.

\section{RESULTADOS}

Houve grande prevalência do modelo cinestésico para os estudantes de Odontologia e do auditivo para os de Psicologia, ambos superando a metade de cada amostra estudada, conforme descrito na Tabela 1.

\begin{tabular}{|ccccc|}
\hline Curso de graduação & \multicolumn{4}{c|}{ Estilo de aprendizagem (VARK) } \\
\cline { 2 - 5 } (amostra) & Visual (V) & Auditivo (A) & Leitura/Escrita(R) & Cinestésico (K) \\
\hline Odontologia $(n=49)$ & $8,16 \%$ & $38,78 \%$ & $14,29 \%$ & $51,02 \%$ \\
Psicologia $(n=47)$ & $14,89 \%$ & $74,47 \%$ & $8,51 \%$ & $27,66 \%$
\end{tabular}

Tabela 1 - Frequência relativa dos estilos de aprendizagem (VARK) de estudantes da Saúde Fonte: elaborada pelos autores (2020).

Os estudantes de Psicologia tiveram pontuações menores do que os de Odontologia para as dimensões fisiológica, segurança, social e estima. Quanto à realização pessoal, ambos os 
grupos foram similares. Foi destaque a baixa frequência de condições satisfatórias de acesso à internet na Psicologia, o que pode representar um desafio para atividades on-line. Para os estudantes que apresentaram pontuações abaixo de 4 em todas as dimensões, foi recomendado adicionalmente um acompanhamento social ou psicoterapêutico individual pelos setores especializados da própria instituição. As medianas resultantes de cada item, segundo a dimensão por curso, foram expressas na Figura 2.

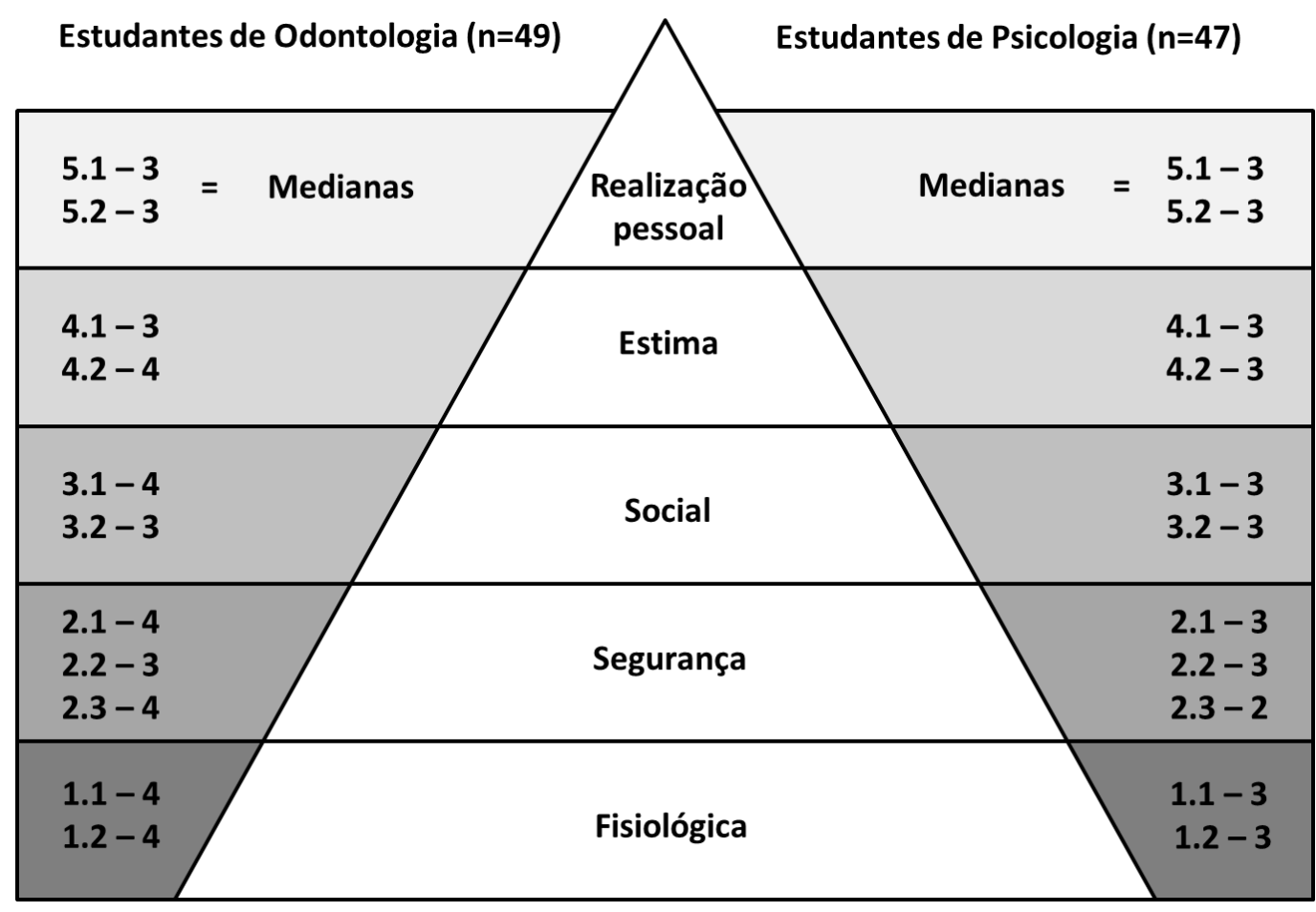

Figura 2 - Comparação da hierarquia de necessidades humanas de Maslow em estudantes da Saúde na pandemia

Fonte: elaborada pelos autores (2020).

As atividades teóricas prosseguiram no curso de Odontologia e foram interrompidas no curso de Psicologia, por decisão colegiada e do corpo estudantil até o retorno oficial do calendário acadêmico, o que explica acessibilidade aquém da observada na Odontologia, conforme demonstra o Gráfico 1. 


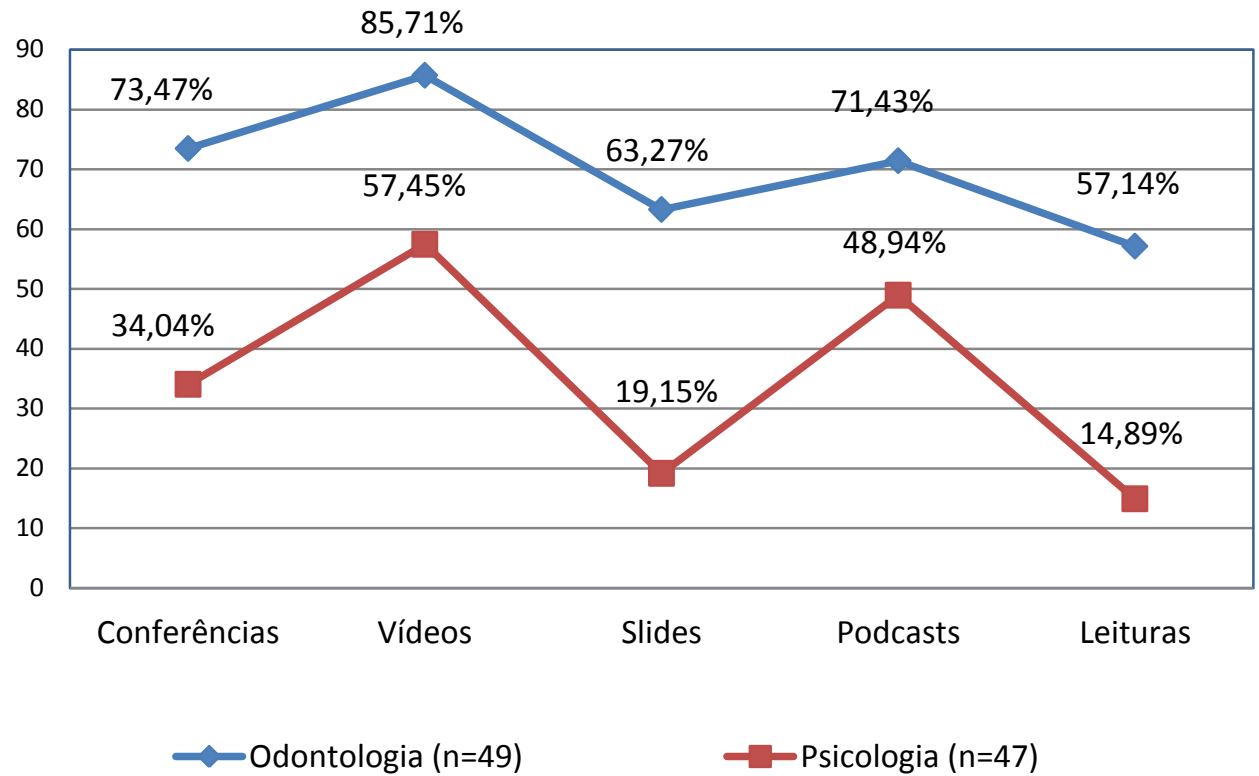

Gráfico 1 - Análise do acesso de estudantes da Saúde aos recursos remotos na pandemia Fonte: elaborado pelos autores (2020).

Foi possível observar correlação entre o estilo de aprendizagem VARK com o tipo de recurso digital acessado. Vídeos assíncronos, conferências síncronas e podcasts foram recursos mais acessados pelos estudantes dos cursos de Odontologia e de Psicologia, o que é compatível com os perfis de aprendizagem cinestésico e auditivo, respectivamente. Slides e leituras tiveram menor aderência, mas ainda assim reforçam sua importância frente à baixa dimensão de segurança de Maslow observada, na qual o ambiente educacional com recursos assíncronos possibilita maior inclusão digital, em especial para o curso de Psicologia.

De acordo com a frequência de citações sobre os recursos didáticos multimídias utilizados, foi possível observar que percepções positivas foram mais prevalentes em ambos os cursos, convergindo nas expressões "boa" e "dinâmica". Mesmo no curso de Psicologia, em que as opiniões foram mais heterogêneas em relação à Odontologia, observou-se a prevalência de expressões positivas frente às percepções de insatisfação, conforme demonstram as Figuras 3 e 4. 


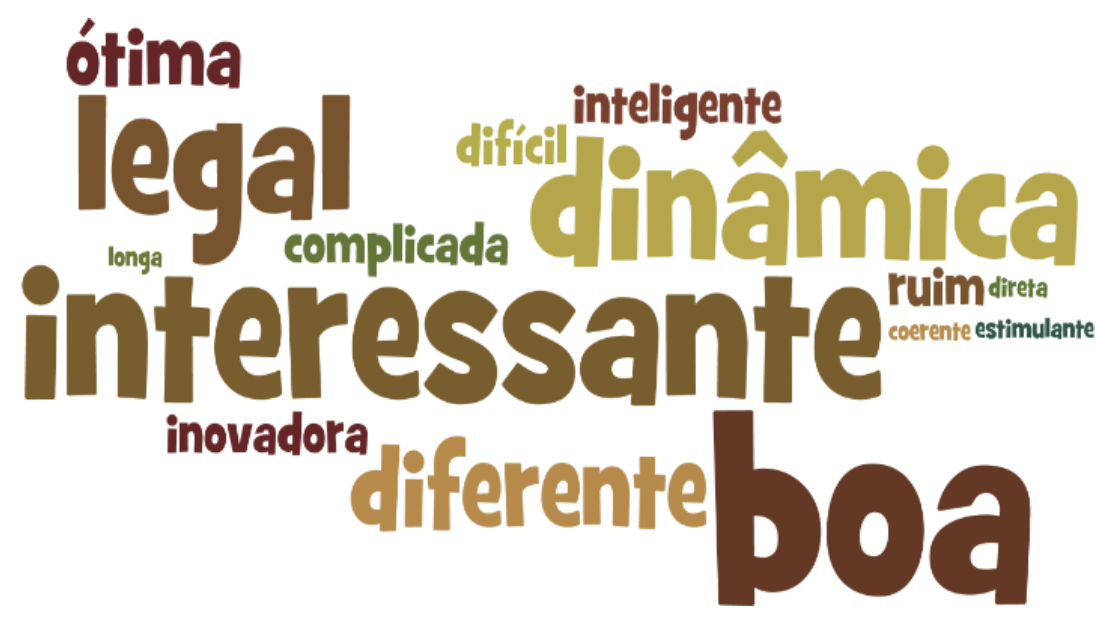

Figura 3 - Nuvem de palavras com representação das percepções de estudantes de Odontologia sobre aulas remotas na pandemia $(n=49)$

Fonte: elaborada pelos autores (2020).

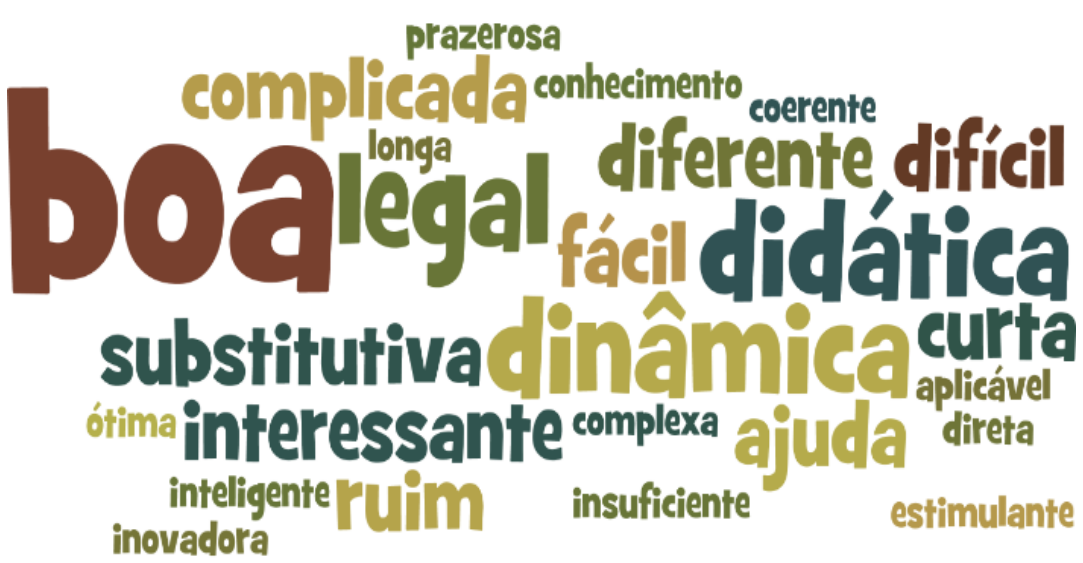

Figura 4 - Nuvem de palavras com representação das percepções de estudantes de Psicologia sobre aulas remotas na pandemia $(n=47)$

Fonte: elaborada pelos autores (2020).

\section{DISCUSSÃO}

O Ceará configura como o oitavo estado com mais instituições de ensino superior atuantes em EaD (ABED, 2019) e exibiu a terceira maior incidência em COVID-19 do país no primeiro trimestre da pandemia deflagrada em março de 2020, com franca disseminação para cidades do interior (MACIEL; CASTRO-SILVA; FARIAS, 2020). O processo educacional no distanciamento social pela pandemia é um desafio sem precedentes para docentes da área de Saúde, atores que buscam novas estratégias diante de um cenário incerto (RAIMONDI, 2020). As instituições de ensino buscam desenvolver as boas habilidades de professores e tutores a distância, que incluem comunicação, conteúdo, promoção de aprendizagem e 
Igor luco Castro-Silva, Jacques Antonio Cavalcante Maciel, Lana Karine Araújo

empatia (ABED, 2019). Esforços alinham-se ao propósito de minimizar o baixo desempenho e a evasão acadêmica, frequentes em primeiros semestres da graduação (CASTRO-SILVA; GUERRERO; MACIEL, 2018).

Estudos sobre estilos de aprendizagem usando a tipologia VARK demonstram que estudantes de Odontologia no México podem exibir o modelo visual maior que a auditivo e cinestésico (JIMÉNEZ ORTIZ et al., 2019), enquanto estudantes de Psicologia no Brasil se associam mais a um perfil auditivo, seguido do cinestésico (CASTRO-SILVA; GUERRERO; MACIEL, 2018). O presente relato corrobora o esperado para a Psicologia, mas o perfil cinestésico foi mais prevalente para a Odontologia, contrariando a literatura. Este fenômeno poderia ser creditado às competências profissionais desenvolvidas em cada curso da Saúde (SANTOS, V. et al., 2020), em que estudantes de Odontologia estariam mais envolvidos com habilidades técnicas procedurais, enquanto estudantes de Psicologia, com a escuta ativa e qualificada.

Bolsas acadêmicas podem contribuir de forma mais imediata para o enfrentamento de desigualdades de acesso à educação superior, como observado em estudantes de Medicina de uma universidade pública, onde bolsistas tiveram melhor desempenho, reforçando a importância de programas de apoio à manutenção dos estudos (MOREIRA et al., 2019). Sob um enfoque bastante peculiar, as instituições com EaD no Brasil autodeclaram pouca oferta de atendimento humano destinado à acessibilidade digital, o que pode representar um viés (ABED, 2019). Ainda, o cuidado com a saúde física e mental do estudante merece ser uma política institucional permanente (DIAS et al., 2020). Tais evidências mostram que o combate às diferentes formas de iniquidade digital, do ponto de vista financeiro, profissional e pessoal, é importante para minimizar impactos do ambiente educacional sobre a ensinagem remota em tempos de pandemia (ZAYAPRAGASSARAZAN, 2020). O questionário de necessidades humanas proposto pelos autores mostrou-se de fácil aplicação e pode ser generalizável para outros cursos, da Saúde e de outras áreas, para diagnóstico preliminar de aspectos de vulnerabilidade dos estudantes. A interpretação dos fatores modificáveis referentes às cinco dimensões de Maslow merece um olhar amiúde das instituições de ensino para favorecerem um ambiente educacional inclusivo (TRONCON, 2014).

Estratégias didáticas, que articulem teoria e prática, acompanham as mudanças pedagógicas da educação superior das profissões da Saúde e contam com diferentes tecnologias de informação e comunicação (TICs) (SANTOS, J. et al., 2018). Um estudo da Associação Brasileira de Educação a Distância com instituições de educação superior do país demostrou que a participação de graduandos em tutorias baseadas em videoaulas e videoconferências superou a adesão de redes sociais (ABED, 2019). Contudo, o uso de Facebook em disciplina de Saúde Coletiva ajudou a integrar a formação médica dos estudantes com a educação em saúde comunitária (BERNARDES et al., 2019). De forma similar, o Whatsapp ambientou uma 
Igor luco Castro-Silva, Jacques Antonio Cavalcante Maciel, Lana Karine Araújo

discussão dinâmica longitudinal durante um semestre letivo sobre conteúdos de Promoção de Saúde, Educação em Saúde, Prevenção de Agravos e Determinantes Sociais, gerando alta satisfação entre os discentes (PAULINO et al., 2018). Podcasts unem parte da teoria cognitiva de aprendizagem multimídia, pelo uso de expressão verbal ou memória sensorial, à andragogia, baseada no conhecimento prévio ou estratégias mnemônicas de longo prazo, tendo alta validade como recurso aplicável na educação superior (MCNAMARA; DREW, 2019). A sala de aula invertida potencializa a aprendizagem ativa e colaborativa para profissões da Saúde, apesar de inerentes limitações técnicas, que incluem qualidade ruim, gravações longas, dificuldades de facilitação no trabalho em grupo e acesso aos recursos mínimos de informática para os estudantes acompanharem demandas pré-classe (BOLLELA, 2017). Tais experiências observadas na literatura temática sobre TICs corroboram a opção por videoaulas, conferências e podcasts neste relato, reforçando a hipótese de que a forma assíncrona ofereceria um modelo digital mais inclusivo para atividades remotas.

Cabe salientar que outras TICs podem ser valorosas na ensinagem em Saúde, isoladas ou em associação. O protótipo de um aplicativo móvel pode contribuir para o treinamento de equipe multiprofissional de saúde na prevenção de erros de medicação (CALDAS et al., 2020). Portfólio virtual constitui ferramenta reflexiva preciosa, estimulante da visão crítica e autoavaliação do processo educativo, com aplicações da graduação até a pós-graduação (CASTRO-SILVA; MACIEL, 2019). Uso de blog acadêmico promoveu melhoria nos domínios psicomotor e afetivo em estudantes de Odontologia, apesar desta ferramenta estar em franco desuso diante de novas mídias digitais videoassistidas (VASCONCELOS et al., 2016). Ainda, metodologias ativas típicas da forma presencial, como problematização para pequenos grupos de estudantes (MACIEL et al., 2016) e aprendizagem em equipes para grandes grupos (CASTRO-SILVA; GUERRERO; MACIEL, 2018; CASTRO-SILVA; MACIEL, 2017), poderiam unir-se às TICs para engajar estudantes durante a pandemia (ZAYAPRAGASSARAZAN, 2020). Todas as metodologias que fomentem uma aprendizagem ausubeliana, significativa, que favoreça a interação do binômio facilitador-aprendiz e trace estratégias mnemônicas por subsunçores, são positivas para a ensinagem, cabendo também reconhecer, além de suas potencialidades, as limitações inerentes a cada cenário educacional (CASTRO-SILVA; MACIEL, 2017).

\section{CONSIDERAÇÕES FINAIS}

Os estilos de aprendizagem VARK e a hierarquia de necessidades humanas de Maslow podem contribuir positivamente para o planejamento pedagógico de atividades teóricas a distância em estudantes de Odontologia e Psicologia. É importante promover a divulgação desses instrumentos para uso em outros cursos superiores da Saúde. 
Recursos digitais cinestésicos e auditivos, incluindo vídeos e podcasts, sugerem maior envolvimento dos discentes de ambos os cursos. A oferta assíncrona de conteúdos pode minimizar limitações de acessibilidade digital ou do ambiente educacional verificadas entre os estudantes de um campus de universidade pública do interior do Ceará.

Este relato de experiência teve como limitações a pequena amostra, elencada de acordo com a governabilidade dos docentes das disciplinas elencadas, e o curto tempo de acompanhamento, mediante a necessidade de enfrentamento à pandemia. Supervisão longitudinal dos estudantes da Saúde é relevante para compreensão do reflexo de metodologias e recursos digitais de ensinagem remota no contexto pandêmico brasileiro.

\section{AGRADECIMENTOS}

Ao Governo do Estado do Ceará e à Fundação Cearense de Apoio ao Desenvolvimento Científico e Tecnológico (Processo BP3-0139-00270.01.00/18 - Edital BPI 03/2018), pelo apoio à pesquisa.

\section{REFERÊNCIAS}

ABED - ASSOCIAÇÃO BRASILEIRA DE EDUCAÇÃO A DISTÂNCIA. Censo EaD.BR: relatório analítico da aprendizagem a distância no Brasil 2018. Curitiba: InterSaberes, 2019.

BERNARDES, Viviane Pereira et al. Facebook como ferramenta pedagógica em saúde coletiva: integrando formação médica e educação em saúde. Revista Brasileira de Educação Médica, Brasília, v. 43, n. 1, p. 652-661, 2019. DOI: http://dx.doi.org/10.1590/19815271v43suplemento1-20190192.

BOLLELA, Valdes Roberto. Sala de aula invertida na educação para as profissões de Saúde: conceitos essenciais para a prática. Revista Eletrônica de Farmácia, Goiânia, v. 14, n. 1, p. 3948, 2017. DOI: https://doi.org/10.5216/ref.v14i1.42807.

BRASIL. Presidência da República. Lei n. 9.394, de 20 de dezembro de 1996. Estabelece as diretrizes e bases da educação nacional. Diário Oficial da União, Brasília, DF. 23 dez. 1996.

BRASIL. Ministério da Educação. Portaria n. 544, de 16 de junho de 2020. Dispõe sobre a substituição de aulas presenciais por aulas em meios digitais. Diário Oficial da União, Brasília, DF. 17 jun. 2020.

CALDAS, Marciele Misiak et al. Aplicativo móvel para prevenção de erros de medicação: Prevmed. Ciencia y enfermería, Concepción, v. 26, n. 4, p. 1-9, 2020. DOI: http://dx.doi.org/10.4067/s0717-95532020000100401.

CASTRO-SILVA, Igor luco; GUERRERO, Jesus Alberto Perez; MACIEL, Jacques Antonio Cavalcante. De cromossomos a como somos: ressignificando o estudo de Fisiologia para 
Igor luco Castro-Silva, Jacques Antonio Cavalcante Maciel, Lana Karine Araújo

Psicologia. In: DICKMANN, Ivanio. (org.). DNA Educação 3. São Paulo: Dialogar, 2018. p. 107125.

CASTRO-SILVA, Igor luco; MACIEL, Jacques Antonio Cavalcante. Avaliação educacional com aprendizagem baseada em equipes em um curso de Odontologia. In: CIASCA, Maria Isabel Filgueiras Lima et al. (org.). Avaliação e seus espaços: desafios e reflexões. Fortaleza: UFC, 2017. p. 1287-1303.

CASTRO-SILVA, Igor luco; MACIEL, Jacques Antonio Cavalcante. Portfólio: uma ferramenta de avaliação formativa para o stricto sensu. Indagatio Didactica, Aveiro, v. 11, n. 3, p. 167-179, 2019. DOI: https://doi.org/10.34624/id.v11i3.4942.

DIAS, Lineker Fernandes et al. Promoção da saúde: coerência nas estratégias de ensinoaprendizagem. Revista Brasileira de Educação Médica, Brasília, v. 43, n. 1, p. 641-651, 2020. https://doi.org/10.1590/1981-5271v43suplemento1-20190104.

JIMÉNEZ ORTIZ, José Leonardo et al. Identificación de Estilos de Aprendizaje en Estudiantes de Odontología en México mediante el Modelo VARK. Revista Educación en Ciencias de la Salud, Concepción, v. 16, n. 1, p. 6-9, 2019.

MACIEL, Jacques Antonio Cavalcante et al. A integração ensino-serviço em Odontologia: uma experiência na Atenção Primária à Saúde no município de Sobral. Revista de APS - Atenção Primária à Saúde, Juiz de Fora, v. 19, n. 4, p. 650-655, 2016.

MACIEL, Jacques Antonio Cavalcante; CASTRO-SILVA, Igor luco; FARIAS, Mariana Ramalho. Análise inicial da correlação espacial entre a incidência de COVID-19 e o desenvolvimento humano nos municípios do estado do Ceará no Brasil. Revista Brasileira de Epidemiologia, Rio de Janeiro, v. 23, 2020. DOI: https://doi.org/10.1590/1980-549720200057.

MACIEL, Jacques Antonio Cavalcante; JIMENEZ, Haydee Gina Quispe; CASTRO-SILVA, Igor luco. Percepções de médicos cubanos sobre o processo de trabalho na Atenção Primária à Saúde no Ceará, Brasil. SODEBRAS, Guaratinguetá, v. 15, n. 175, p. 41-46, 2020. DOI: https://doi.org/10.29367/issn.1809-3957.15.2020.175.41.

MCNAMARA, Scott; DREW, Christopher. Concept analysis of the theories used to develop educational podcasts. Educational Media International, Londres, v. 56, n. 4, p. 300-312, 2019. DOI: https://doi.org/10.1080/09523987.2019.1681107.

MOREIRA, Glaucia de Oliveira et al. Desempenho acadêmico de estudantes bolsistas durante o curso de Medicina. Revista Brasileira de Educação Médica, Brasília, v. 43, n. 3, p. 163-169, 2019. DOI: https://doi.org/10.1590/1981-52712015v43n3rb20180180.

PANÚNCIO-PINTO, Maria Paula; TRONCON, Luiz Ernesto de Almeida. Avaliação do estudante: aspectos gerais. Medicina, Ribeirão Preto, v. 47, n. 3, p. 314-323, 2014. DOI:

https://doi.org/10.11606/issn.2176-7262.v47i3p314-323. 
Igor luco Castro-Silva, Jacques Antonio Cavalcante Maciel, Lana Karine Araújo

PAULINO, Danilo Borges et al. WhatsApp como recurso para Educação em Saúde: contextualizando teoria e prática em um novo cenário de ensino-aprendizagem. Revista Brasileira de Educação Médica, Brasília, v. 42, n. 1, p. 170-178, 2018. DOI: https://doi.org/10.1590/1981-52712018v42n1rb20170061.

PROBST, Carla Fátima Romanoski. (Trad.) Questionário VARK [Internet]. Disponível em: https://vark-learn.com/questionario/. Acesso em: 23 jul. 2020.

RAIMONDI, Gustavo Antonio. Between applauses and loneliness, heroes/warriors and fear: thoughts of a medical professor during the COVID-19 pandemic. Qualitative Inquiry, Londres, 2020. DOI: https://doi.org/10.1177/1077800420941051.

SANTOS, José Luís Guedes et al. Estratégias didáticas no processo de ensino-aprendizagem de gestão em Enfermagem. Texto \& Contexto Enfermagem, Florianópolis, v. 27, n. 2, 2018. DOI: http://dx.doi.org/10.1590/0104-070720180001980016R.

SANTOS, Victor Hugo et al. Currículo oculto, educação médica e profissionalismo: uma revisão integrativa. Interface, Botucatu, v. 24, 2020. DOI: https://doi.org/10.1590/interface.190572.

SCHMITT, Camila da Silva; DOMINGUES, Maria José Carvalho de Souza. Estilos de aprendizagem: um estudo comparativo. Avaliação, Campinas, v. 21, n. 2, p. 361-385, 2016. DOI: http://dx.doi.org/10.1590/S1414-40772016000200004.

SILVA, Welinton Baxto da; AMARO, Rosana; MATTAR, João. Distance education and the open university of Brazil: history, structure, and challenges. The International Review of Research in Open and Distributed Learning, Athabasca, v. 20, n. 4, p. 99-115, 2019. DOI: https://doi.org/10.19173/irrodl.v20i4.4132.

TRONCON, Luiz Ernesto de Almeida. Ambiente educacional. Medicina, Ribeirão Preto, v. 47, n. 3, p. 264-271, 2014. DOI: https://doi.org/10.11606/issn.2176-7262.v47i3p264-271.

VASCONCELOS Jann Lucca Apolonio et al. Blog como ferramenta integrada ao processo ensino-aprendizagem em curso de Odontologia. Brazilian Journal of Surgery and Clinical Research, Cianorte, v. 16, n. 1, p. 12-17, 2016.

ZAYAPRAGASSARAZAN, Zayabalaradjane. COVID-19: strategies for engaging remote learners in medical education. F1000 Research, Londres, v. 9, 2020. DOI: https://doi.org/10.7490/f1000research.1117835.1. 


\section{Igor luco Castro-Silva}

Bacharel em Odontologia (UFF), especialista em Gestão de Organização Pública de Saúde (UNIRIO), mestre em Patologia (UFF) e doutor em Odontologia (UFF). Professor dos cursos de Odontologia e Psicologia (UFC Sobral). Docente permanente do mestrado acadêmico em Biotecnologia (UFC Sobral), de Didática do Ensino Superior e Bioética. Líder do Grupo de Estudos em Morfologia (DGP-CNPq). Bolsista de produtividade (FUNCAP).

igor.iuco@sobral.ufc.br

\section{Jacques Antonio Cavalcante Maciel}

Bacharel em Odontologia (UFC), especialista em Política, Planejamento, Gestão e Avaliação em Saúde Bucal (UFPE), mestre em Saúde da Família e Doutor em Odontologia (UFC). Professor do curso de Odontologia (UFC Sobral). Docente do mestrado profissional da Rede Nordeste de Formação em Saúde da Família/Universidade Estadual Vale do Acaraú e do mestrado acadêmico em Saúde da Família da UFC. Líder do Núcleo de Epidemiologia e Pesquisa Interprofissional em Saúde Coletiva.

jacques.maciel@sobral.ufc.br

\section{Lana Karine Araújo}

Bacharela em Odontologia (UFC) e mestranda em Biotecnologia pela Universidade Federal do Ceará, Campus de Sobral (UFC). Áreas de atuação: Estágio de Docência I, Clínica odontológica e Biomateriais.

lanakarine1380@gmail.com 